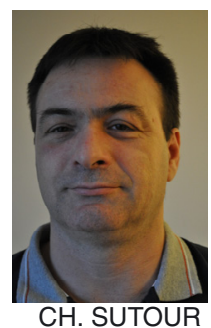

\title{
Développement d'un matériau de référence certifié de formaldéhyde sur cartouche de DNPH
}

\section{Development of formaldehyde certified reference material on DNPH cartridge}

\author{
Christophe SUTOUR, Julien GRENOUILLET, Tatiana MACÉ et Sophie VASLIN-REIMANN \\ Laboratoire national de métrologie et d'essais (LNE), 1 rue Gaston Boissier, 75015 Paris, France, christophe.sutour@lne.fr.
}

\section{Résumé}

En climat tempéré, la population passe, en moyenne, $85 \%$ de son temps dans des environnements clos, et une majorité de ce temps dans l'habitat. Différents types de sources (mobilier, sols, murs, plafonds...) peuvent être à l'origine de la présence de polluants dans ces environnements clos. Pour faire face à l'enjeu sanitaire que représente la qualité de l'air intérieur et apporter aux pouvoirs publics des éléments utiles à la gestion de ce risque, l'Agence nationale de sécurité sanitaire de l'alimentation, de l'environnement et du travail (Anses) travaille depuis 2004 à l'élaboration de valeurs guides de qualité d'air intérieur (VGAI), fondées exclusivement sur des critères sanitaires. Depuis le début de ce travail, l'Anses a identifié onze polluants d'intérêt de l'air intérieur dont le formaldéhyde connu pour ses effets irritants. Depuis 2004, le formaldéhyde est aussi classé par le Centre international de recherche sur le cancer (CIRC) comme «substance cancérogène avérée pour l'homme » (groupe 1) et depuis 2007 fait l'objet de VGAI (VGAI à court terme de $50 \mu \mathrm{g} \cdot \mathrm{m}^{-3}$ pour une durée d'exposition de deux heures et des VGAI à long terme de $10 \mu \mathrm{g} \cdot \mathrm{m}^{-3}$ pour une durée d'exposition supérieure à un an). De nombreuses campagnes de mesures sont régulièrement réalisées, notamment dans les logements, les établissements recevant du public dont les écoles ou les crèches, mais aussi dans des lieux atypiques comme les piscines, les gymnases, etc. L'objectif de cette étude était donc de développer des matériaux de référence certifiés (MRC) se présentant sous la forme de cartouches imprégnées de 2,4-dinitrophénylhydrazine (DNPH) contenant une quantité connue de formaldéhyde sur une gamme de masses comprises entre $1 \mu \mathrm{g}$ et $10 \mu \mathrm{g}$ correspondant aux masses communément prélevées dans le domaine de l'air intérieur, et ce pour assurer la traçabilité des mesures de formaldéhyde réalisées par les laboratoires d'analyse. Pour atteindre cet objectif, le LNE a mis au point un banc permettant la génération de formaldéhyde gazeux basé sur la mise en œuvre de tubes à perméation d'alpha-polyoxyméthylène et une méthode de chargement des cartouches DNPH. La caractérisation de cette méthode conduit à des résultats de mesure ayant des incertitudes finales relatives sur les masses de formaldéhyde comprises entre $3,3 \%$ et $8 \%$ avec une stabilité de 20 jours pour une gamme de masses comprises entre $1 \mu \mathrm{g}$ et $10 \mu \mathrm{g}$; cette méthode met également en évidence les influences notables de certains paramètres comme la stabilité dans le temps, des cartouches chargées en formaldéhyde.

MOTS CLÉS : FORMALDÉHYDE, CARTOUCHE DNPH, MATÉRIAU DE RÉFÉRENCE CERTIFIÉ.

\begin{abstract}
In temperate climate, people spend on average $85 \%$ of their time in closed environments and a majority of that time in housing. Different sources (furniture, floors, walls, ceilings) may be responsible for the presence of pollutants in these closed environments. To address the health challenge raised by the quality of indoor air and provide useful elements for the management of this risk to public authorities, the French agency for food, environmental and occupational health and safety (Anses) works since 2004 in the development of indoor air guideline values (IAGVs), based exclusively on health criteria. Since the beginning of this work, Anses identified eleven indoor air pollutants of interest including formaldehyde known for its irritant effects. Since 2004, formaldehyde is classified by the International Agency for Research on Cancer (IARC) as "proven carcinogen to humans" (Group 1) and since 2007 is subject to IAGVs (short term IAGVs $50 \mu \mathrm{g} \cdot \mathrm{m}^{-3}$ for an exposure of two hours and long term IAGVs $10 \mathrm{ug} \cdot \mathrm{m}^{-3}$ for an exposure of more than one year). Many measurement campaigns are regularly carried out, particularly in housing, public buildings including schools and childcare facilities, but also in unusual places such as swimming pools, gymnasiums, etc. The objective of this study was to develop Certified Reference Materials (CRMs) which are in the form of cartridges impregnated with 2,4-dinitrophenylhydrazine (DNPH) containing a known amount of formaldehyde over a mass range of $1 \mu \mathrm{g}$ to $10 \mu \mathrm{g}$ corresponding to masses commonly measured in the field of indoor air, and to ensure the traceability of formaldehyde measurements performed by the analytical laboratories. To achieve this, LNE has developed a bench allowing the generation of formaldehyde gas mixtures based on the use of permeation tubes of alpha-polyoxymethylene and a method
\end{abstract}


of loading DNPH cartridges. The characterization of this method leads to final uncertainties measurements on formaldehyde masses ranging from $3.3 \%$ to $8 \%$ and a stability of 20 days for a mass range from $1 \mu \mathrm{g}$ to $10 \mu \mathrm{g}$; it also highlights significant influence of some parameters such as the stability over the time on formaldehyde masses loaded on the cartridges.

KEY WORDS: FORMALDEHYDE, DNPH CARTRIDGE, CERTIFIED REFERENCE MATERIAL.

\section{Contexte}

En climat tempéré, la population passe en moyenne $85 \%$ de son temps dans des environnements clos, et une majorité de ce temps dans l'habitat, source de pollution.

Depuis quelques années, une attention croissante est portée à ce sujet, avec en particulier la création par les pouvoirs publics, en 2001, de l'Observatoire de la qualité de l'air intérieur (OQAI). Cet observatoire a organisé de nombreuses campagnes de mesures dans les environnements intérieurs et a notamment publié en 2006 les résultats d'une campagne nationale de la qualité de l'air réalisée dans 567 logements [1]. L'interprétation des résultats de ce type de mesures nécessite de disposer de valeurs repères qui permettent de positionner les niveaux observés par rapport à des critères sanitaires.

Par conséquent, pour faire face à l'enjeu sanitaire que représente la qualité de l'air intérieur et apporter aux pouvoirs publics des éléments utiles à la gestion de ce risque, l'Agence nationale de sécurité sanitaire de l'alimentation, de l'environnement et du travail (Anses) travaille à l'élaboration de valeurs guides de qualité d'air intérieur (VGAI), fondées exclusivement sur des critères sanitaires.

Depuis le début de ce travail, onze polluants d'intérêt de l'air intérieur dont le formaldéhyde $\left(\mathrm{CH}_{2} \mathrm{O}\right)$ ont été étudiés et ont fait l'objet d'une expertise de l'Anses sur les VGAI. La VGAI à court terme fixée en 2007 par l'Anses pour le formaldéhyde est de $50 \mu \mathrm{g} \cdot \mathrm{m}^{-3}$ pour une exposition de deux heures; la VGAI à long terme fixée en $2007 \mathrm{est}$, elle, de $10 \mu \mathrm{g} \cdot \mathrm{m}^{3}$ pour une exposition supérieure à un an [2].

De plus, depuis 2004, le formaldéhyde est classé par le Centre international de recherche sur le cancer (CIRC) comme «substance cancérogène avérée pour l'homme » (groupe 1).

Les campagnes de mesure réalisées pour évaluer les niveaux de formaldéhyde en air intérieur sont basées sur le prélèvement du formaldéhyde par pompage de l'air ambiant à travers une cartouche imprégnée de 2,4dinitrophénylhydrazine (DNPH) qui réagit par dérivation avec les molécules d'intérêt. Ces cartouches sont ensuite analysées par des laboratoires d'analyse : le composé est alors extrait avec de l'acétonitrile et analysé par chromatographie liquide haute performance (HPLC) puis détecté dans l'UV à $360 \mathrm{~nm}$. Le chromatographe peut être étalonné avec une solution commerciale de formaldéhyde (DNPH dans l'acétonitrile). Néanmoins, cette méthode d'étalonnage ne permet pas d'intégrer la phase d'extraction que subit le formaldéhyde complexé dans la cartouche imprégnée de DNPH.

\section{Objectif de l'étude}

Dans ce cadre, l'objectif de cette étude était donc de développer des MRC se présentant sous la forme de cartouches imprégnées de DNPH contenant une quantité connue de formaldéhyde sur une gamme de masses comprises entre $1 \mu \mathrm{g}$ et $10 \mu \mathrm{g}$ correspondant aux masses communément prélevées dans le domaine de l'air intérieur pour assurer la traçabilité des mesures de formaldéhyde réalisées par les laboratoires d'analyse.

Pour atteindre cet objectif, le LNE a mis au point un banc permettant la génération de formaldéhyde gazeux et une méthode de chargement des cartouches DNPH.

\section{Description de la méthode de chargement et d'analyse des cartouches}

\subsection{Principe de la méthode de chargement}

Des cartouches contenant de la 2,4dinitrophénylhydrazine (DNPH) sont chargées par balayage avec un mélange gazeux de formaldéhyde généré par la méthode de perméation. Lors du chargement, le formaldéhyde réagit avec la DNPH contenue dans les cartouches pour former un hydrazone selon la réaction de dérivation décrite en figure 1. La DNPH est en excès jusqu'à des masses chargées de formaldéhyde de $75 \mu \mathrm{g}$ (spécifications données par le fabricant SUPELCO). Dans notre cas (chargement de $1 \mu \mathrm{g}$ à $10 \mu \mathrm{g}$ ), la réaction est donc totale, tout le formaldéhyde est dérivé. Cette dérivation permet une détection dans l'UV qui n'est pas possible avec la molécule de formaldéhyde seule.

À partir du taux de perméation du tube de formaldéhyde et en fonction du temps pendant lequel le chargement est effectué, la masse de formaldéhyde déposée peut être calculée. Cette méthode de chargement a été utilisée pour réaliser l'ensemble des essais présentés dans cet article.

\subsection{Description du banc de chargement des cartouches et de génération du formaldéhyde}

Le banc de chargement des cartouches de DNPH en formaldéhyde est constitué d'une source de formaldéhyde basée sur la perméation gazeuse régulée en température dans un four VICI ${ }^{\circledR}$, de régulateurs de débit massique Bronkhorst ${ }^{\circledR}$ qui régulent le flux d'azote $(99,9999 \%)$ entrainant les molécules de formaldéhyde et d'une vanne d'injection quatre voies VICI traitée Silcosteel ${ }^{\circledR}$ reliée à la cartouche de DNPH à charger (fig. 2). Les tuyauteries du système sont traitées Silcosteel ${ }^{\circledR}$ afin de les rendre inertes et les volumes morts ont été réduits au maximum afin d'éviter toute fuite ou adsorption. 


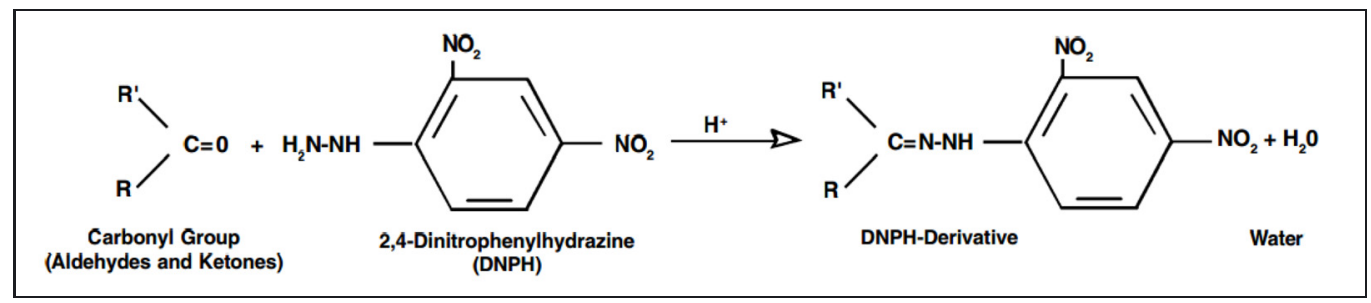

Fig. 1. - Réaction de dérivation des aldéhydes par la DNPH.

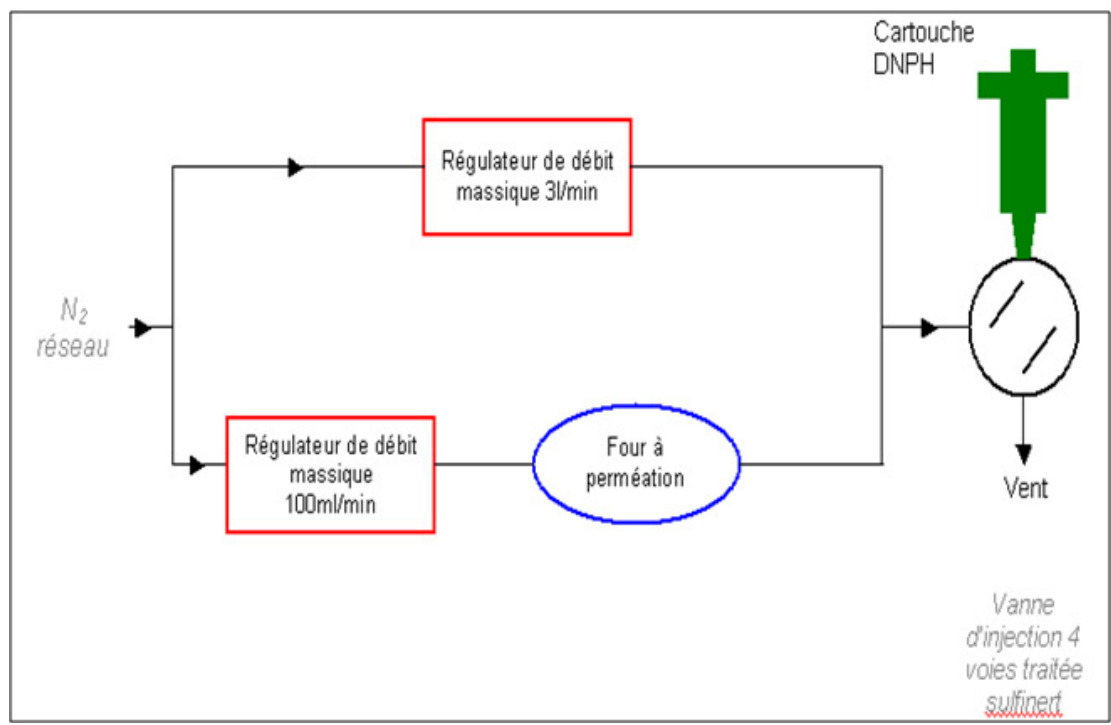

Fig. 2. - Schéma de principe du banc de génération du formaldéhyde et de chargement des cartouches DNPH.

Le four à perméation (dynacalibrator) régulé en température à $(80 \pm 0,01)^{\circ} \mathrm{C}$ possède des parois internes rendues inertes chimiquement par un traitement des surfaces (Silcosteel $\left.{ }^{\circledR}\right)$. Il contient un tube à perméation d'alphapolyoxyméthylène commercialisé par la société Kin-Tek qui est balayé lors du chargement des cartouches en formaldéhyde par un débit d'azote total de $1,3 \mathrm{~L} \cdot \mathrm{min}^{-1}$ (débit optimum pour le chargement des cartouches DNPH dans les conditions normales de pression et température). Afin de connaître la quantité de formaldéhyde libérée par le tube à perméation au cours du temps, le tube est pesé mensuellement.

L'alpha-polyoxyméthylène lors de sa sublimation va libérer du formaldéhyde mais également de l'eau. La perte de masse mesurée par pesée correspond donc à la somme des pertes de masse de formaldéhyde et d'eau. Il est alors nécessaire de corriger le taux de perméation calculé initialement en fonction de la concentration en vapeur d'eau présente dans le gaz.

\subsection{Justification des choix techniques}

\subsubsection{Choix de la méthode de génération du formaldéhyde gazeux}

Plusieurs méthodes permettent de générer du formaldéhyde gazeux.
Le LNE possède un système d'évaporation de liquide contrôlé appelé CEM (Bronkhorst), constitué d'un régulateur de débit liquide, d'un régulateur de débit massique pour le gaz vecteur et d'une chambre de mélange/évaporation régulée en température. Ce système nécessite l'utilisation du composé pur en phase liquide pour y être vaporisé, et ainsi générer le mélange gazeux de référence. Le formaldéhyde ne se trouve que sous forme de mélange liquide à environ $37 \%$ dans de l'eau. L'utilisation de ce type de solution n'est pas compatible avec notre application (présence d'eau et pas de traçabilité de ces solutions). Par conséquent, ce principe n'a pas été retenu.

Une autre solution envisagée était d'utiliser des mélanges de gaz préparés par le LNE avec la méthode gravimétrique. Néanmoins, le mode d'introduction du composé formaldéhyde pose problème, car il se trouve soit en solution liquide soit sous forme de solide. De plus, il n'existe pas à ce jour de bouteille de gaz pourvu d'un traitement interne permettant une stabilité suffisante du mélange gazeux dans le temps.

Une troisième méthode consiste à générer des mélanges gazeux de façon dynamique par perméation : cette méthode est utilisée au LNE pour différents composés depuis plus de vingt ans. La maitrise des pesées et la connaissance de la pureté du composé pur permettent de générer des mélanges gazeux stables avec de faibles incertitudes. 
Pour toutes ces raisons, le LNE a choisi d'utiliser un système de perméation pour la génération des mélanges gazeux de référence en formaldéhyde.

\subsubsection{Choix du composé pur pour le tube à perméation}

La génération de formaldéhyde par perméation consiste en un tube en téflon rempli d'un composé pur dont la membrane laisse passer une certaine quantité de gaz à une température donnée.

Plusieurs composés purs sont utilisables pour générer le formaldéhyde gazeux à savoir le paraformaldéhyde et l'alpha-polyoxyméthylène.

Ces deux composés sont des polymères. Ils sont identiques dans la structure, mais la longueur de la chaîne carbonée $(n)$ de l'alpha-polyoxyméthylène est plus longue $(100<n<300$ contre $8<n<100$ pour le paraformaldéhyde), ce qui lui confère une meilleure pureté puisqu'il y a formation d'une molécule d'eau pour $\mathrm{n}$ molécules de formaldéhyde.

Afin de comparer ces deux types de sources de formaldéhyde, des concentrations de l'ordre de $350 \mathrm{nmol} \cdot \mathrm{mol}^{-1}$ de formaldéhyde dans l'azote ont été générées avec ces deux sources et analysées à l'aide d'un spectromètre laser (QCL-TILDAS) de marque Aerodyne, dont la quantification est déterminée à partir de la base de données spectrale (HITRAN).

Les résultats obtenus ont montré des écarts relatifs entre la mesure de la concentration effectuée par le spectromètre laser et la concentration générée par perméation de l'ordre de $0,1 \%$ pour une source d'alphapolyoxyméthylène et de $-2,5 \%$ pour une source de paraformaldéhyde. Ces résultats montrent une meilleure cohérence entre les analyses réalisées par spectrométrie laser et la concentration théorique générée par perméation à partir d'une source d'alpha-polyoxyméthylène.

Le LNE a donc choisi d'utiliser un tube à perméation contenant de l'alpha-polyoxyméthylène et de le réguler à une température de $80{ }^{\circ} \mathrm{C}$ dans un four permettant ainsi sa dépolymérisation en formaldéhyde.

\subsubsection{Choix du four à perméation}

Le tube à perméation doit être placé dans un «puits » dont les parois sont totalement inertes vis-à-vis du formaldéhyde. Ce puits doit être régulé en température et balayé en continu par un flux gazeux. L'innocuité avec les parois du puits et la qualité de la régulation de température (10\% par degré) sont des facteurs très importants pour la stabilité du taux de perméation et de la génération d'un mélange gazeux par perméation qui doit être juste et stable.

Le four à perméation $\mathrm{VICI}{ }^{\circledR}$ remplit ces critères puisqu'il régule la température à $\pm 0,01{ }^{\circ} \mathrm{C}$ et possède un traitement interne des surfaces qui le rend inerte chimiquement.

Une analyse comparative avec le spectromètre laser entre le gaz de balayage prélevé en amont du four et le gaz de balayage prélevé en aval du four sans présence de tube à perméation conduit à des concentrations pour le gaz de balayage en amont et en aval du four inférieures à la limite de détection évaluée à $1 \mathrm{nmol} \cdot \mathrm{mol}^{-1}$. L'innocuité du four à perméation est donc vérifiée.

\subsubsection{Choix du composé pur pour le tube à perméation}

L'objectif de cette étude étant de pouvoir fournir des matériaux de référence certifiés (MRC) pour le formaldéhyde, il convenait de développer ces MRC avec un type de cartouches le plus couramment utilisé d'une part, par les laboratoires d'analyse pour réaliser l'étalonnage de leurs appareils et d'autre part, par les Associations Agréées de Surveillance de la Qualité de l'Air (AASQA) pour effectuer leurs prélèvements sur site afin de limiter des biais éventuels et d'utiliser les mêmes méthodes d'extraction.

Les cartouches les plus couramment utilisées étant des cartouches Lp DNPH de marque Supelco ${ }^{\circledR}$, il a été décidé de développer des MRC sur ce type de cartouches.

\subsection{Choix de la méthode d'analyse des cartouches}

L'objectif du LNE était de développer et de caractériser la méthode de chargement des cartouches et non de développer une méthode d'analyse du formaldéhyde faisant déjà l'objet de la norme NF ISO 16000-3 qui consiste à effectuer une désorption chimique de la cartouche par percolation d'acétonitrile (quelques millilitres) : l'éluant obtenu est ensuite injecté (sous forme liquide) dans une chromatographie liquide haute performance (HPLC) puis analysé dans l'UV à 360 nm.

De ce fait, pour réaliser les analyses, il a été choisi un laboratoire accrédité Cofrac (Airparif). Une pré-étude a été effectuée en amont pour valider le choix du laboratoire. Les résultats des essais préliminaires étant conformes au cahier des charges du LNE, le choix du laboratoire a pu être validé et toutes les analyses des cartouches DNPH afférentes à cette étude ont été réalisées par ce laboratoire.

\section{Détermination de la masse de formaldéhyde chargée sur les cartouches et de l'incertitude associée}

\subsection{Calcul de la masse de formaldéhyde chargée}

La masse de formaldéhyde déposée sur la cartouche se calcule à partir du taux de perméation du tube et du temps pendant lequel le mélange gazeux traverse la cartouche. Cependant, un certain nombre de corrections listées ci-après sont ajoutées afin de tenir compte de facteurs d'influence.

$$
m_{\mathrm{CH}_{2} \mathrm{O}}=T_{x} \times R \times 10^{-3} \times t_{\mathrm{c}}+C_{\text {blanc }}+C_{\text {répé }}+C_{\text {stab }}+C_{\text {trans }},
$$

avec :

$m_{\mathrm{CH}_{2} \mathrm{O}}$ la masse de formaldéhyde chargée sur la cartouche $(\mu \mathrm{g})$; 
$T_{x} \quad$ le taux de perméation du tube $\left(\mathrm{ng} \cdot \mathrm{min}^{-1}\right)$;

$R \quad$ le rendement de chargement (sans dimension);

$t_{\mathrm{c}} \quad$ la durée du chargement de la cartouche (min);

$C_{\text {blanc }}$ la correction liée à la présence de formaldéhyde dans les blancs des cartouches neuves $(\mu \mathrm{g})$;

$C_{\text {répé }}$ la correction liée à la répétabilité du chargement $(\mu \mathrm{g})$;

$C_{\text {stab }} \quad$ la correction liée à la stabilité dans le temps des masses de formaldéhyde chargées sur les cartouches $(\mu \mathrm{g})$;

$C_{\text {trans }}$ la correction liée au transport sur la masse chargée $(\mu \mathrm{g})$.

\subsection{Estimation de l'incertitude élargie sur la masse de formaldéhyde chargée}

La variance sur la masse de formaldéhyde chargée est déterminée en utilisant la loi de propagation des variances [3] dont la formule générale pour $y=f\left(x_{i}, x_{j}\right)$ est :

$$
\begin{aligned}
u_{c}^{2}(y)= & \sum_{i=1}^{N}\left(\frac{\partial f}{\partial x_{\mathrm{i}}}\right)^{2} \times u^{2}\left(x_{i}\right) \\
& +2 \sum_{i=1}^{N-1} \sum_{j=i+1}^{N}\left(\frac{\partial f}{\partial x_{\mathrm{i}}}\right)\left(\frac{\partial f}{\partial x_{\mathrm{j}}}\right) u\left(x_{i}, x_{j}\right) .
\end{aligned}
$$

Si l'on applique cette loi à l'équation (1), on obtient :

$$
\begin{aligned}
u^{2}\left(m_{\mathrm{CH}_{2} \mathrm{O}}\right)= & \left(R \times 10^{-3} \times t_{C}\right)^{2} \times u^{2}\left(T_{x}\right) \\
& +\left(T_{x} \times 10^{-3} \times t_{C}\right)^{2} \times u^{2}(R) \\
& +\left(T_{x} \times R \times 10^{-3}\right)^{2} \times u^{2}\left(t_{\mathrm{c}}\right) \\
& +\left(\frac{\partial m_{\mathrm{CH}_{2} \mathrm{O}}}{\partial C_{\text {blanc }}}\right)^{2} \times u^{2}\left(C_{\text {blanc }}\right) \\
& +\left(\frac{\partial m_{\mathrm{CH}_{2} \mathrm{O}}}{\partial C_{\text {répé }}}\right)^{2} \times u^{2}\left(C_{\text {répé }}\right) \\
& +\left(\frac{\partial m_{\mathrm{CH}_{2} \mathrm{O}}}{\partial C_{\text {stab }}}\right)^{2} \times u^{2}\left(C_{\text {stab }}\right) \\
& +\left(\frac{\partial m_{\mathrm{CH}_{2} \mathrm{O}}}{\partial C_{\text {trans }}}\right)^{2} \times u^{2}\left(C_{\text {trans }}\right) .
\end{aligned}
$$

L'incertitude élargie $U(k=2)$ sur la masse de formaldéhyde chargée sur les cartouches est donnée par l'équation (2):

$$
U\left(m_{\mathrm{CH}_{2} \mathrm{O}}\right)=2 \times \sqrt{u^{2}\left(m_{\mathrm{CH}_{2} \mathrm{O}}\right)} .
$$

Les paragraphes ci-après décrivent les démarches suivies pour estimer chacune de ces variances.

\section{Estimation de la pureté du tube à perméation}

\subsection{Objectif}

Le taux de perméation correspond à la quantité de matière traversant une paroi perméable pendant un temps donné. La détermination du taux de perméation s'effectue par pesée du tube à perméation à intervalles de temps réguliers (de l'ordre d'un mois).

Pour déterminer la valeur du taux de perméation, outre la perte de masse du tube au cours du temps et différentes autres corrections, il est nécessaire de connaître la pureté du composé d'intérêt, et donc de développer en amont une procédure permettant de l'estimer, ainsi que l'incertitude associée.

Les publications traitant de la dépolymérisation ou de la dégradation thermique de l'alpha-polyoxyméthylène sont unanimes quant à la génération de formaldéhyde et d'eau comme produits de dépolymérisation ou de dégradation thermique [4-11]. À la température d'utilisation du système, soit $80^{\circ} \mathrm{C}$, et selon Biesecker [5] et Duan [7], la vitesse de dépolymérisation ou de la dégradation thermique est très faible. Le risque de former des produits de dégradation complexes est donc limité, puisque les réactions de scission du polymère se font à température élevée.

Le LNE a donc décidé d'orienter l'étude de la quantification des impuretés en sortie du tube à perméation sur l'impureté majoritaire, à savoir l'eau.

\subsection{Problématique}

La source de formaldéhyde utilisée étant un tube à perméation, les concentrations générées par celui-ci avec un gaz de dilution d'azote à $1,3 \mathrm{~L} \cdot \mathrm{min}^{-1}$, sont de l'ordre de quelques centaines de $\mathrm{nmol} \cdot \mathrm{mol}^{-1}$ de formaldéhyde et les traces de vapeur d'eau de quelques $\mathrm{nmol} \cdot \mathrm{mol}^{-1}$.

Pour réaliser les analyses, il est donc nécessaire de disposer d'un appareil de mesure de vapeur d'eau avec une limite de détection très faible $\left(\mathrm{nmol} \cdot \mathrm{mol}^{-1}\right)$, et un système de mise en œuvre des gaz exempt de toute fuite.

\subsection{Choix des matériels}

\subsubsection{Système de perméation}

Au vu des difficultés liées à la détermination de la concentration en vapeur d'eau émise par le tube à perméation, il a été décidé de ne pas faire cette détermination à l'aide des fours à perméation habituellement utilisés.

Un montage spécifique a été réalisé à partir d'un réservoir en acier inoxydable électropoli muni de raccords VCR garantissant une très bonne étanchéité et donc l'absence de contamination extérieure. Un système de chauffage avec régulation PID a été ajouté pour permettre une régulation en température de l'ensemble. 


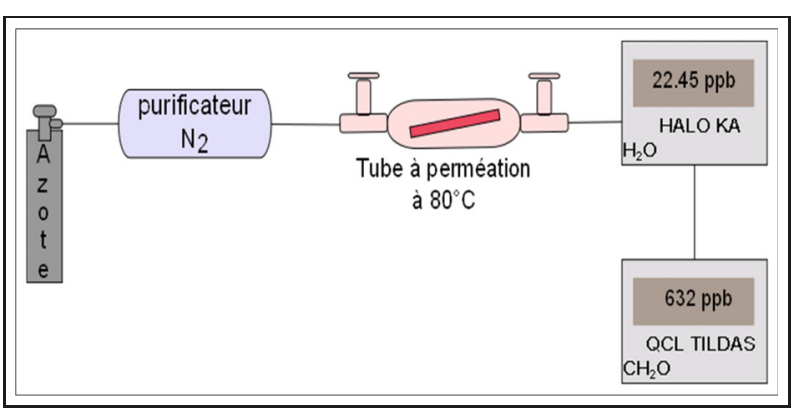

Fig. 3. - Montage pour la détermination des impuretés en vapeur d'eau.

Le réservoir en acier inoxydable utilisé a l'avantage de pouvoir recevoir plusieurs tubes à perméation afin d'augmenter la concentration émise en formaldéhyde et donc en vapeur eau. Le système a donc été équipé de quatre tubes à perméation permettant de générer des concentrations de formaldéhyde de l'ordre de $1500 \mathrm{nmol} \cdot \mathrm{mol}^{-1}$.

\subsubsection{Analyseurs}

Les mesures de concentrations en formaldéhyde ont été réalisées à l'aide du spectromètre laser QCL-TILDAS (spectrométrie d'absorption) de la société Aerodyne. Le spectromètre est constitué d'un laser continu émettant à une longueur d'onde de 5,67 $\mu \mathrm{m}$, d'un détecteur MCT (tellurure de mercure-cadmium) et d'une cellule d'absorption de trajet optique $76 \mathrm{~m}$.

Les mesures de concentration en vapeur d'eau sont réalisées à l'aide d'un spectromètre laser HALO-KA (spectrométrie «CRDS ») de la société Tiger Optics. Ce spectromètre mesure le temps d'extinction de la lumière dans une cavité optique (trajet de plusieurs dizaines de kilomètres) à deux longueurs d'onde : l'une où la vapeur d'eau absorbe la lumière (1 $392,5 \mathrm{~nm})$ et l'autre où elle n'absorbe pas.

Ces deux appareils reposent sur des principes de mesure optique garantissant des mesures absolues (base de données HITRAN). Leurs limites de détection sont inférieures à $1 \mathrm{nmol} \cdot \mathrm{mol}^{-1}$ et l'incertitude relative sur la mesure est de $5 \%$, ce qui convient parfaitement à notre problématique.

\subsection{Principe et caractérisation du système analytique mis en œuvre}

\subsubsection{Principe}

Le schéma de principe est donné sur la figure 3.

De l'azote pur $(99,9999 \%)$ est filtré $\left(\mathrm{H}_{2} \mathrm{O}<\right.$ $\left.0,1 \mathrm{nmol} \cdot \mathrm{mol}^{-1}\right)$ au moyen d'un filtre Entegris et circule à un débit de $1 \mathrm{~L} \cdot \mathrm{min}^{-1}$ dans le réservoir contenant les tubes à perméation maintenus à une température de $80{ }^{\circ} \mathrm{C}$ par une couverture chauffante régulée par PID

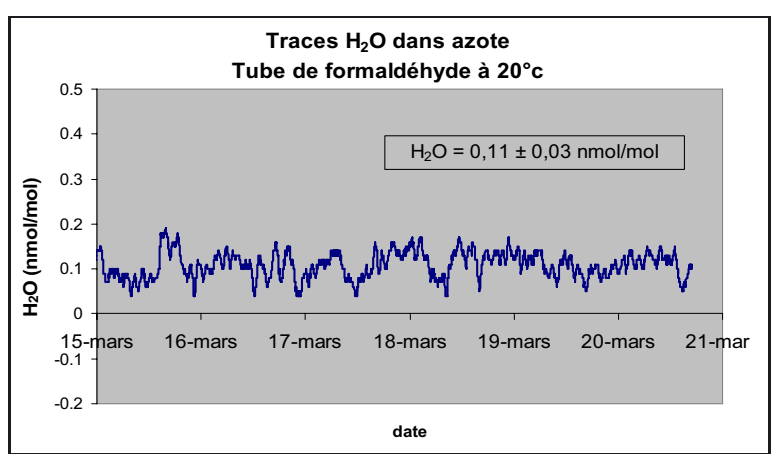

Fig. 4. - Évolution des concentrations en vapeur d'eau à $20^{\circ} \mathrm{C}$.

(Proportionnelle, Intégrale et Dérivée). Le mélange gazeux (azote + formaldéhyde + eau) est analysé de manière simultanée afin de quantifier la vapeur d'eau et le formaldéhyde contenus dans ce mélange gazeux.

\subsubsection{Détermination du bruit de fond}

La température du four contenant les tubes à perméation est réglée à $20^{\circ} \mathrm{C}$ et la concentration en vapeur d'eau est analysée pendant six jours afin de connaître le bruit de fond du système (blanc).

Les résultats montrent une très bonne stabilité des mesures et un niveau de concentration en vapeur d'eau très faible $\left(0,11 \mathrm{nmol} \cdot \mathrm{mol}^{-1}\right)$ (fig. 4$)$.

Par conséquent, l'azote balayant les tubes à perméation est exempt de traces de vapeur d'eau et le circuit fluidique n'apporte pas de contamination au regard de la résolution de l'analyseur de vapeur d'eau.

\subsection{Détermination de la concentration relative molaire en $\mathrm{H}_{2} \mathrm{O}$}

\subsubsection{Principe}

La concentration relative molaire en vapeur d'eau $C_{\% \mathrm{H}_{2} \mathrm{O}}\left(10^{-2} \mathrm{~mol} \cdot \mathrm{mol}^{-1}\right)$ est calculée à partir de la moyenne des mesures effectuées.

$$
C_{\% \mathrm{H}_{2} \mathrm{O}}=100 \times \frac{\left(C_{\mathrm{H}_{2} \mathrm{O}}+j_{\mathrm{H}_{2} \mathrm{O}}\right)}{\left(C_{\mathrm{H}_{2} \mathrm{O}}+j_{\mathrm{H}_{2} \mathrm{O}}\right)+\left(C_{\mathrm{CH}_{2} \mathrm{O}}+j_{\mathrm{CH}_{2} \mathrm{O}}\right)}
$$

avec :

- $C_{\mathrm{H}_{2} \mathrm{O}}$ la concentration en vapeur d'eau mesurée $\left(\mathrm{nmol} \cdot \mathrm{mol}^{-1}\right)$;

- $j_{\mathrm{H}_{2} \mathrm{O}}$ la justesse de la mesure en vapeur d'eau $\left(\mathrm{nmol} \cdot \mathrm{mol}^{-1}\right)$;

- $C_{\mathrm{CH}_{2} \mathrm{O}}$ la concentration en formaldéhyde mesurée $\left(\mathrm{nmol} \cdot \mathrm{mol}^{-1}\right)$;

- $j_{\mathrm{CH}_{2} \mathrm{O}}$ la justesse de la mesure en formaldéhyde $\left(\mathrm{nmol} \cdot \mathrm{mol}^{-1}\right)$.

Cette équation implique de déterminer les concentrations en formaldéhyde et en vapeur d'eau et les incertitudes associées (cf. paragraphes ci-après). 


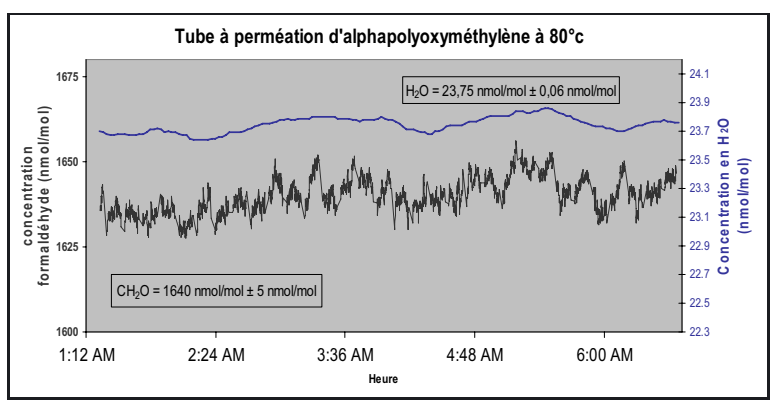

Fig. 5. - Évolution des concentrations en formaldéhyde et en vapeur d'eau à $80^{\circ} \mathrm{C}$.

L'incertitude élargie $(k=2)$ sur la justesse des mesures sera considérée comme étant égale à $5 \%$ de la concentration mesurée pour le formaldéhyde conformément à l'étude effectuée par le LNE dans le cadre du Laboratoire Central de Surveillance de la Qualité de l'Air (LCSQA) de novembre 2012 [12] et pour la vapeur d'eau conformément à la publication de Hisashi [13].

\subsubsection{Résultats obtenus}

La température du four est réglée à une consigne de $80{ }^{\circ} \mathrm{C}$. La concentration en vapeur d'eau dans l'air ambiant est de l'ordre de $1 \times 10^{-2} \mathrm{~mol} \cdot \mathrm{mol}^{-1}$. Les molécules de vapeur d'eau s'adsorbent très facilement sur les parois internes des tuyauteries. Il est donc très difficile de mesurer des traces de vapeur d'eau. Une décontamination des tuyauteries est alors nécessaire pendant plusieurs semaines pour atteindre la stabilisation des mesures en raison des faibles valeurs de concentration mesurée.

Après cette phase de stabilisation, les concentrations en formaldéhyde et en vapeur d'eau ont été mesurées en sortie du réservoir contenant les tubes à perméation (fig. 5).

Le suivi de l'évolution des concentrations dans le temps met en évidence certaines fluctuations liées à des variations de température des tubes. Il est important de noter que ces tendances sont visibles de manière simultanée sur les concentrations en formaldéhyde et en vapeur d'eau ce qui témoigne de la corrélation entre les deux puisque la dépolymérisation de l'alphapolyoxyméthylène conduit à la formation de molécules de formaldéhyde et de vapeur d'eau. De plus, une augmentation de la température entraîne une augmentation du taux de perméation du tube de formaldéhyde, de sa concentration, ainsi que de la concentration en vapeur d'eau.

La figure 5 met également en évidence la qualité des analyseurs utilisés, puisque les écarts types relatifs sont inférieurs à $0,5 \%$.

Malgré les variations des concentrations mesurées sur cinq jours différents et liées aux fluctuations de température du système, le rapport des concentrations est relativement constant et témoigne de la corrélation existante
Tableau 1

Détermination de la reproductibilité sur la pureté.

\begin{tabular}{|c|c|c|c|}
\hline Date & $\begin{array}{c}\text { Concentration } \\
\text { en } \mathrm{CH}_{2} \mathrm{O} \\
\left(\mathrm{nmol} \cdot \mathrm{mol}^{-1}\right)\end{array}$ & $\begin{array}{c}\text { Concentration } \\
\text { en } \mathrm{H}_{2} \mathrm{O} \\
\left(\mathrm{nmol} \cdot \mathrm{mol}^{-1}\right)\end{array}$ & $\begin{array}{c}\text { Concentration } \\
\text { relative en } \\
\mathrm{H}_{2} \mathrm{O}(\%)\end{array}$ \\
\hline $05 / 12 / 2014$ & 1511 & 21,60 & 1,41 \\
\hline $19 / 05 / 2014$ & 1610 & 23,11 & 1,42 \\
\hline $20 / 05 / 2014$ & 1640 & 23,75 & 1,43 \\
\hline $22 / 05 / 2014$ & 1363 & 18,96 & 1,37 \\
\hline $23 / 05 / 2014$ & 1368 & 19,12 & 1,38 \\
\hline
\end{tabular}

entre la concentration de formaldéhyde et celle en vapeur d'eau (tab. 1).

Ces essais conduisent à une concentration relative en vapeur d'eau $C_{\%} \mathrm{H}_{2} \mathrm{O}$ de : $(1,40 \pm 0,22) \times 10^{-2} \mathrm{~mol} \cdot \mathrm{mol}^{-1}$ $(k=2)$.

L'incertitude sur la concentration relative en vapeur d'eau est la somme quadratique des variances sur la reproductibilité des mesures et de l'incertitude sur chacune des déterminations.

\subsection{Calcul de la pureté massique}

La pureté en formaldéhyde, $\alpha$, doit être calculée en rapport massique afin de pouvoir corriger le taux de perméation du tube $\left(\mathrm{ng} \cdot \mathrm{min}^{-1}\right)$ déterminé à partir de sa perte de masse en fonction du temps. Celle-ci s'exprime de la façon suivante :

$$
\alpha=\frac{1}{\left.1+\frac{C_{\%_{2}} \mathrm{O}}{\left(1-C_{\%_{2} \mathrm{O}} \mathrm{O}\right.}\right)} \times \frac{M_{\mathrm{H}_{2} \mathrm{O}}}{M_{\mathrm{CH}_{2} \mathrm{O}}},
$$

avec :

- $\alpha$, la pureté

- $C_{\% \mathrm{H}_{2} \mathrm{O}}$, la concentration relative molaire en vapeur d'eau ;

- $M_{\mathrm{H}_{2} \mathrm{O}}$, la masse molaire de l'eau $\left(\mathrm{g} \cdot \mathrm{mol}^{-1}\right)$;

- $M_{\mathrm{CH}_{2} \mathrm{O}}$, la masse molaire du formaldéhyde $\left(\mathrm{g} \cdot \mathrm{mol}^{-1}\right)$.

Le rapport massique en formaldéhyde $\alpha$ déterminée en tenant compte de l'analyse des traces de vapeur d'eau est égale à $0,9916 \pm 0,0014(k=2)$.

Ces valeurs seront prises en compte dans le calcul du taux de perméation du tube et de l'incertitude associée.

\section{Caractérisation de la méthode de chargement des cartouches}

Toutes les analyses de formaldéhyde sur cartouches DNPH ont été réalisées par le laboratoire d'analyses accrédité COFRAC d'Airparif. Les incertitudes relatives sur les masses analysées sont de $10 \%(k=2)$ et la limite de quantification est $0,09 \mu \mathrm{g}$. Ces valeurs ont été déterminées par le laboratoire d'Airparif lors de la validation de leur méthode. 


\subsection{Rendement de chargement}

Une cartouche de DNPH a été balayée par un mélange gazeux de formaldéhyde à environ $224 \mathrm{nmol} \cdot \mathrm{mol}^{-1}$. La concentration en formaldéhyde en sortie de la cartouche a été mesurée en continu avec le spectromètre laser QCLTILDAS jusqu'à saturation de cette dernière (au bout de 2 h 45 environ).

Les concentrations de formaldéhyde mesurées pendant les 2 h 45 sont inférieures à la limite de détection du spectromètre laser. Ces résultats montrent donc que la totalité du formaldéhyde traversant la cartouche de DNPH reste absorbée, même après plus de deux heures de chargement.

Le rendement du chargement sera considéré comme étant égal à 1 avec une incertitude de $0,0052(k=2)$ faisant intervenir principalement la limite de détection du spectromètre.

\subsection{Masse de formaldéhyde des «blancs » des cartouches DNPH}

La masse de formaldéhyde analysée sur des cartouches non exposées à du formaldéhyde (blanc) peut légèrement varier d'une cartouche à l'autre. Dans la mesure où chaque cartouche est à usage unique, il n'est pas possible de connaître la masse de formaldéhyde contenue dans chaque cartouche neuve.

Afin de tenir compte de ce biais dans le calcul de la masse de formaldéhyde chargée sur une cartouche, 41 analyses de cartouches neuves ont été réalisées au cours des différents essais de cette étude. Il en résulte une masse moyenne de formaldéhyde de 0,036 $4 \mu \mathrm{g}$, avec un écart type de reproductibilité de $0,024 \mu \mathrm{g}$ (Norme NF ISO 5725-1 [14]).

L'incertitude type sur la masse en formaldéhyde contenue sur une cartouche vierge $u\left(C_{\text {blanc }}\right)$ est :

$$
u\left(C_{\text {blanc }}\right)=\sqrt{\left(\frac{\sigma_{\text {repro }}}{\sqrt{n}}\right)^{2}+\left(u_{\text {analyse }}\right)^{2}},
$$

avec :

- $\sigma_{\text {repro }}$, écart type de reproductibilité des blancs ;

- $u_{\text {analyse }}$, incertitude sur l'analyse des cartouches égale à $10 \%(k=2)$.

Soit

$$
u\left(C_{\text {blanc }}\right)=\sqrt{\left(\frac{0,024}{\sqrt{41}}\right)^{2}+\left(\frac{0,0036}{2}\right)^{2}}
$$

ce qui conduit à :

$$
C_{\text {blanc }}=(0,0364 \pm 0,0042) \mu \mathrm{g}(k=1) .
$$

Tableau 2

Influence de la matrice (azote).

\begin{tabular}{|c|c|c|c|}
\hline \multicolumn{2}{|c|}{ Chargement avec de l'azote } & \multicolumn{2}{c|}{ Sans chargement } \\
\hline Cartouche & $\begin{array}{c}\text { Masse de } \\
\text { formaldéhyde } \\
\text { analysée }(\mu \mathrm{g})\end{array}$ & Cartouche & $\begin{array}{c}\text { Masse de } \\
\text { formaldéhyde } \\
\text { analysée }(\mu \mathrm{g})\end{array}$ \\
\hline 1 & 0,06 & 6 & 0,04 \\
\hline 2 & 0,05 & 7 & 0,03 \\
\hline 3 & 0,06 & 8 & 0,04 \\
\hline 4 & 0,04 & 9 & 0,04 \\
\hline 5 & 0,05 & 10 & 0,08 \\
\hline Moyenne & 0,05 & - & 0,05 \\
\hline
\end{tabular}

\subsection{Influence de la matrice}

Afin d'évaluer l'effet de la matrice sur les cartouches, cinq cartouches ont été chargées avec le gaz de dilution (azote) pendant $11 \mathrm{~min}$ à un débit de $1500 \mathrm{~mL} \cdot \mathrm{min}^{-1}$ (conditions de chargement pour $5 \mu \mathrm{g}$ ) puis analysées en les comparant à cinq cartouches sans chargement. Les résultats obtenus sont synthétisés dans le tableau 2.

Ces résultats ne montrent pas de différence significative entre des cartouches vierges et des cartouches balayées par de l'azote. Par conséquent, le gaz de dilution (azote) n'apporte pas de contamination lors du chargement des cartouches.

\subsection{Débit de chargement optimum et influence sur la masse chargée}

La masse de formaldéhyde chargée sur les cartouches dépend du taux de perméation du tube et du temps de chargement (aux corrections près).

Le constructeur préconise un débit optimum de chargement de $1500 \mathrm{~mL} \cdot \mathrm{min}^{-1}$. Le LNE a vérifié que ce débit de chargement est adapté à la réalisation de matériaux de référence certifiés, et s'est assuré que la variation du débit de chargement autour du débit optimum n'avait pas d'influence sur la masse finale de formaldéhyde chargée.

Quinze cartouches ont donc été chargées à environ $5 \mu \mathrm{g}$ avec des débits de $1,3 \mathrm{~L} \cdot \mathrm{min}^{-1}, 1,5 \mathrm{~L} \cdot \mathrm{min}^{-1}$ et $1,7 \mathrm{~L} \cdot \mathrm{min}^{-1}$.

Les incertitudes mentionnées correspondent aux incertitudes analytiques données par le laboratoire d'analyses d'Airparif.

Quels que soient les débits de chargement $\left(1,3 \mathrm{~L} \cdot \mathrm{min}^{-1}, 1,5 \mathrm{~L} \cdot \mathrm{min}^{-1}\right.$ et $\left.1,7 \mathrm{~L} \cdot \mathrm{min}^{-1}\right)$, la masse de formaldéhyde chargée reste identique aux incertitudes près (tab. 3). La variation du débit de chargement autour de la valeur optimale préconisée par le constructeur n'a donc pas d'influence significative sur la masse chargée.

\subsection{Répétabilité de la méthode}

Afin de déterminer la répétabilité de la méthode de chargement des cartouches, le LNE a chargé trois séries de dix cartouches à des masses de $1 \mu \mathrm{g}, 5 \mu \mathrm{g}$ et $10 \mu \mathrm{g}$ 
Tableau 3

Influence du débit de chargement.

\begin{tabular}{|l|c|c|c|c|c|}
\hline \multicolumn{2}{|c|}{$\begin{array}{c}\text { Chargement } \\
\text { à } 1,3 \mathrm{~L} \cdot \mathrm{min}^{-1}\end{array}$} & \multicolumn{2}{c|}{$\begin{array}{c}\text { Chargement } \\
\text { à } 1,5 \mathrm{~L} \cdot \mathrm{min}^{-1}\end{array}$} & \multicolumn{2}{c|}{$\begin{array}{c}\text { Chargement } \\
\text { à } 1,7 \mathrm{~L} \cdot \mathrm{min}^{-1}\end{array}$} \\
\hline $\begin{array}{l}\text { Masse } \\
\text { analysée, } \\
\text { en } \mu \mathrm{g}\end{array}$ & $\begin{array}{c}U \\
(k=2), \\
\text { en } \mu \mathrm{g}\end{array}$ & $\begin{array}{c}\text { Masse } \\
\text { analysée, } \\
\text { en } \mu \mathrm{g}\end{array}$ & $\begin{array}{c}U \\
(k=2), \\
\text { en } \mu \mathrm{g}\end{array}$ & $\begin{array}{c}\text { Masse } \\
\text { analysée, } \\
\text { en } \mu \mathrm{g}\end{array}$ & $\begin{array}{c}U \\
(k=2), \\
\text { en } \mu \mathrm{g}\end{array}$ \\
\hline 5,23 & 0,52 & 5,20 & 0,52 & 5,19 & 0,52 \\
\hline 5,23 & 0,52 & 5,24 & 0,52 & 5,17 & 0,52 \\
\hline 5,21 & 0,52 & 5,20 & 0,52 & 5,19 & 0,52 \\
\hline 5,20 & 0,52 & 5,19 & 0,52 & 5,22 & 0,52 \\
\hline 5,16 & 0,52 & 5,21 & 0,52 & 5,21 & 0,52 \\
\hline
\end{tabular}

Tableau 4

Répétabilité de la méthode de chargement.

\begin{tabular}{|l|c|c|c|}
\cline { 2 - 4 } \multicolumn{1}{c|}{} & $\begin{array}{c}\text { Écart type de } \\
\text { répétabilité } \\
\text { à } 1 \mu \mathrm{g}\end{array}$ & $\begin{array}{c}\text { Écart type de } \\
\text { répétabilité } \\
\text { à } 5 \mu \mathrm{g}\end{array}$ & $\begin{array}{c}\text { Écart type de } \\
\text { répétabilité } \\
\text { à } 10 \mu \mathrm{g}\end{array}$ \\
\hline${\text { Série } \mathrm{n}^{\circ} 1}^{\circ}$ & 0,011 & 0,038 & 0,030 \\
\hline${\text { Série } \mathrm{n}^{\circ} 2}^{2}$ & 0,043 & 0,038 & 0,026 \\
\hline${\text { Série } \mathrm{n}^{\circ} 3} 3$ & 0,028 & 0,029 & 0,036 \\
\hline $\begin{array}{l}\text { Écart } \\
\text { type } \\
\text { moyen }\end{array}$ & 0,030 & 0,035 & 0,031 \\
\hline
\end{tabular}

soit 90 cartouches Les résultats sont présentés dans le tableau 4.

La répétabilité des mesures a été calculée selon la norme NF ISO 5725-1 [14].

L'incertitude type retenue pour la répétabilité de la méthode de chargement des cartouches de formaldéhyde correspond à l'écart type maximum obtenu précédemment, soit :

$$
u\left(C_{\text {répé }}\right)=0,035 \mu \mathrm{g} \text {. }
$$

\subsection{Stabilité des cartouches chargées}

\subsubsection{Stabilité à court terme}

Des essais de stabilité à court terme ont été réalisés afin de s'assurer que le délai d'analyse du laboratoire n'avait pas d'influence sur la masse de formaldéhyde analysée sur les cartouches, sachant que le laboratoire d'analyses d'Airparif s'était engagé à réaliser les essais au plus tard cinq jours après réception des cartouches.

Le LNE a chargé 12 cartouches à environ $5 \mu \mathrm{g}$ au jour J. L'analyse par lot de trois cartouches a été réalisée les jours 1, 2, 3 et 7 . Les résultats obtenus sont résumés dans le tableau 5.

Les masses de formaldéhyde obtenues à $J+1, J+2$, $J+3$ et $J+7$ ne sont pas significativement différentes. Les cartouches peuvent donc être considérées comme stables entre le moment où elles sont chargées au LNE et le moment où elles sont analysées par le laboratoire.
Tableau 5

Influence du temps d'analyse du laboratoire sur les cartouches chargées à $5 \mu \mathrm{g}$ (stabilité à court terme).

\begin{tabular}{|c|c|c|}
\cline { 2 - 3 } \multicolumn{1}{c|}{} & $\begin{array}{c}\text { Masse analysée, } \\
\text { en } \mu \mathrm{g}\end{array}$ & $\begin{array}{c}\text { Incertitudes élargies } \\
(k=2), \text { en } \mu \mathrm{g}\end{array}$ \\
\hline$J+1$ & 5,43 & 0,53 \\
\hline$J+2$ & 5,45 & 0,53 \\
\hline$J+3$ & 5,44 & 0,53 \\
\hline$J+7$ & 5,46 & 0,53 \\
\hline
\end{tabular}

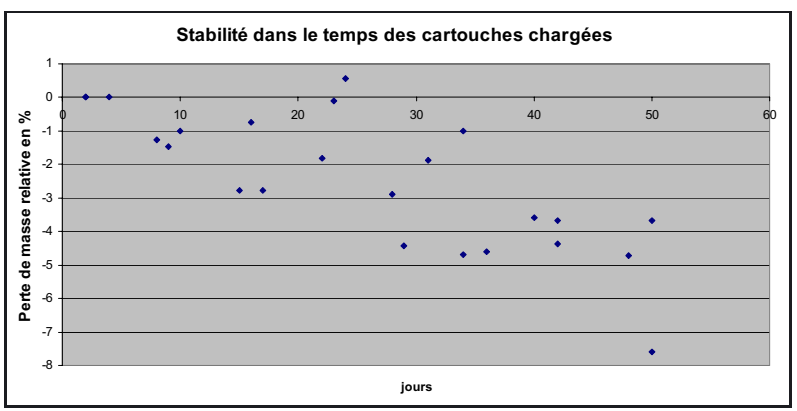

Fig. 6. - Stabilité à long terme des cartouches chargées en formaldéhyde.

\subsubsection{Stabilité à long terme}

Des essais de stabilité des cartouches à plus long terme (jusqu'à 50 jours) ont été réalisés. Pour cela, le LNE a chargé 24 cartouches à des masses de $1 \mu \mathrm{g}, 5 \mu \mathrm{g}$ et $10 \mu \mathrm{g}$.

Les cartouches chargées en formaldéhyde sont stockées au réfrigérateur $\left(4^{\circ} \mathrm{C}\right)$ comme préconisé par le fournisseur de cartouches jusqu'au jour de leur analyse. Les cartouches sont analysées tous les 8 jours sur une période de 50 jours (fig. 6).

Les résultats montrent, même s’ils sont dispersés, une tendance significative à la perte de formaldéhyde sur la cartouche. La modélisation de cette évolution dans le temps permet de la quantifier. La perte de masse relative observée est de 0,097 $1 \%$ de la masse chargée par nombre de jours écoulés après le chargement. Si l'on considère une stabilité des cartouches sur une période de 20 jours après le chargement, il conviendra de considérer une perte de masse relative potentielle 
Tableau 6

Masse de formaldéhyde analysée sans transport et avec transport.

\begin{tabular}{|c|c|c|c|}
\hline \multicolumn{2}{|c|}{$\begin{array}{c}\text { Cartouches non } \\
\text { transportées }\end{array}$} & \multicolumn{2}{|c|}{$\begin{array}{c}\text { Cartouches ayant subi } \\
\text { un transport }\end{array}$} \\
\hline $\begin{array}{c}\text { Masse de } \\
\text { formaldéhyde } \\
\text { analysé } \\
(\mathrm{en} \mu \mathrm{g})\end{array}$ & $\begin{array}{c}U(k=2) \\
(\mathrm{en} \mu \mathrm{g})\end{array}$ & $\begin{array}{c}\text { Masse de } \\
\text { formaldéhyde } \\
\text { analysée } \\
(\mathrm{en} \mu \mathrm{g})\end{array}$ & $\begin{array}{c}U(k=2) \\
(\mathrm{en} \mu \mathrm{g})\end{array}$ \\
\hline 6,00 & 0,61 & 5,97 & 0,60 \\
\hline 6,01 & 0,61 & 5,94 & 0,60 \\
\hline 5,99 & 0,60 & 5,93 & 0,60 \\
\hline 5,97 & 0,60 & 5,96 & 0,60 \\
\hline 5,98 & 0,60 & 5,93 & 0,60 \\
\hline
\end{tabular}

Tableau 7

Bilan des incertitudes pour une masse chargée à $10 \mu \mathrm{g}$.

\begin{tabular}{|l|c|c|c|c|c|c|}
\hline Variable & Unité & Valeur & $u(\mathrm{Xi})$ & $C(\mathrm{Xi})$ & $C(\mathrm{Xi}) u(\mathrm{Xi})$ & Poids relatif $(\%)$ \\
\hline$R$ & & 1,0000 & 0,0026 & 10,2696 & 0,026701 & 2,5 \\
\hline$T_{\mathrm{x}}$ & $\mathrm{ng} \cdot \mathrm{min}^{-1}$ & 427,9 & 3,1 & 0,024 & 0,0744 & 19,2 \\
\hline$t_{\mathrm{c}}$ & $\mathrm{min}$ & 24,00 & 0,020 & 0,4279 & 0,008558 & 0,2 \\
\hline$C_{\text {stab }}$ & $\mu \mathrm{g}$ & 0,00 & 0,11 & 1 & 0,11 & 36,9 \\
\hline$C_{\text {blanc }}$ & $\mu \mathrm{g}$ & 0,0364 & 0,0042 & 1 & 0,0042 & 0,1 \\
\hline$C_{\text {trans }}$ & $\mu \mathrm{g}$ & 0,00 & 0,11 & 1 & 0,11 & 36,9 \\
\hline$C_{\text {répé }}$ & $\mu \mathrm{g}$ & 0,000 & 0,035 & 1 & 0,035 & 4,3 \\
\hline
\end{tabular}

de $2 \%$. Cette erreur potentielle n'étant pas corrigée, elle sera prise comme incertitude type qui, sur une période de 20 jours, s'écrit :

$$
u\left(C_{\text {stab }}\right)=\frac{0,02}{2} \times m_{\mathrm{CH}_{2} \mathrm{O}}=0,01 \times m_{\mathrm{CH}_{2} \mathrm{O}} .
$$

\subsection{Influence du transport sur la masse chargée de formaldéhyde}

Les fabricants des cartouches recommandent dans leur notice d'utilisation de les stocker à une température de $+4{ }^{\circ} \mathrm{C}$. De plus, la norme NF ISO $16000-3$ préconise de réfrigérer les cartouches chargées jusqu'à l'analyse. Cependant, les prélèvements étant rarement effectués au sein même du laboratoire d'analyses, il est important de quantifier l'influence du transport dans le cas où il ne serait pas assuré à la bonne température.

Afin de quantifier l'influence du transport sur la masse chargée, le LNE a chargé dix cartouches dans les locaux du laboratoire d'analyse, cinq d'entre elles n'ont subi aucun transport et les cinq autres ont été transportées dans des boites réfrigérées par un transporteur spécialisé sur une durée de trois jours. Les résultats ont été comparés et sont présentés dans le tableau 6.

Ces essais ont permis de démontrer que le transport des cartouches dans des colis réfrigérés à $4{ }^{\circ} \mathrm{C}$ sur une période de trois jours engendre une légère dégradation de la masse de formaldéhyde $(-0,8 \%)$ contenue dans celleci. L'utilisation du transporteur spécialisé est la solution la plus adaptée pour, à terme, la vente de matériaux de référence certifiés.
Il est donc considéré une incertitude relative liée au transport de $1 \%$ :

$$
u\left(C_{\text {trans }}\right)=0,01 \times m_{\mathrm{CH}_{2} \mathrm{O}} .
$$

\section{Calcul de l'incertitude élargie sur la masse de formaldéhyde chargée}

En se reportant à l'expression de l'incertitude donnée au paragraphe 4.2 et en combinant les différentes incertitudes types calculées en partie 6 , il est évalué des incertitudes élargies relatives comprises entre $3,3 \%$ et $8 \%$ pour des masses de $1 \mu \mathrm{g}$ à $10 \mu \mathrm{g}$ de formaldéhyde déposé sur des cartouches de DNPH.

Les sources d'incertitudes prépondérantes sont : la répétabilité de la méthode, l'effet transport, la stabilité dans le temps et le taux de perméation du tube (exemple d'une masse de $10 \mu \mathrm{g}$ au tab. 7).

\section{Conclusion}

Cette étude a permis de développer un système de génération de formaldéhyde sur la base de tubes à perméation d'alpha-polyoxyméthylène. Ce dispositif a été caractérisé afin de déterminer les différentes sources d'incertitude intervenant sur la concentration de formaldéhyde générée par la source d'alpha-polyoxyméthylène.

La méthode de chargement des cartouches de DNPH développée, permet de proposer des matériaux de référence certifiés (MRC) de formaldéhyde sur de cartouches 
imprégnées de DNPH pour une gamme de masses de $1 \mu \mathrm{g}$ à $10 \mu \mathrm{g}$ avec une durée de stabilité de 20 jours.

La caractérisation de cette méthode a mis en évidence des influences notables de certains paramètres (par exemple, stabilité dans le temps) qui, lorsqu'ils sont combinés selon la loi de propagation des incertitudes, conduisent à une incertitude finale relative sur la masse de formaldéhyde chargée allant de $3,3 \%$ à $8 \%$ sur une gamme de masses de $1 \mu \mathrm{g}$ à $10 \mu \mathrm{g}$.

Les matériaux de référence certifiés pourront être mis en œuvre lors de la validation des méthodes d'analyse des cartouches de DNPH développées par les laboratoires d'analyses et également lors de comparaisons interlaboratoires.

\section{Remerciements}

Les auteurs remercient chaleureusement Julie Gaudin du laboratoire d'analyses chimiques d'Airparif ainsi que son équipe pour leur implication et leur réactivité concernant l'analyse des cartouches de DNPH chargées par le LNE.

\section{Références}

[1] "Premier état de la qualité de l'air dans les logements français", Dossier de presse du 21 novembre 2006 de l'Observatoire de la qualité de l'air intérieur, http://www. oqai.fr/userdata/documents/Document_142.pdf.

[2] "Valeurs guides de qualité d'air intérieur - Le formaldéhyde", AFSSET, juillet 2007.

[3] ISO/IEC Guide 98:2008, "Guide to the expression of uncertainty in measurement”, 2008.

Article reçu le 5 novembre 2015, version révisée reçue le 16 mars 2016.
[4] Hamamoto Masaji, MaEda Seiichi, MiYake Yasuhiko et YANAGISAWA Yoshinari, "Process for producing alphapolyoxymethylene", Brevet, US 3342779 A, publié le 19 septembre 1967.

[5] BIESECKER J.L., "Stabilized powdered formaldehyde", Brevet, US 7799572 B2, publié le 21 septembre 2010.

[6] GRASSIE N. et RochE R.S., Thermal degradation of polyoxymethylene, Die Makromolekulare Chemie, 1968, 112, 16-33.

[7] DUAN Y., Li H., YEL. et al., "Study on the thermal degradation of polyoxymethylene", J. Appl. Polymer Sci., 2006, 99, 6, 3085-3092.

[8] Dudina L.A., Karmilova L.V., Tryapits E.N. et al., "The kinetics and mechanism of thermal and thermaloxidative degradation of formaldehyde polymers", $J$. Polymer Sci. Part C Polymer Symposia, 1967, 2277-2288.

[9] REYNolds R.E. et SLOAN K.T., "Production of monomeric formaldehyde", Brevet, US 3268591 A, publié le 23 août 1966.

[10] DAINTON F.S., Evans D.M., HoARE F.E. et al., "Thermodynamic functions of linear high polymers", Polymer, 1962, 3, 263-321.

[11] Mairesse M., Petit J.M. et Cheron J., "Produits de dégradation thermique des matières plastiques", Cahiers de notes documentaires - Hygiène et sécurité du travail, 1999, 174, 47-57.

[12] Couette J., Grenouillet J., Venault T., Marioni F., Kaiser C., Sutour Ch., MACE T., "Maintien des étalons de référence", Rapport LCSQA, novembre 2012.

[13] ABE H. et YAMADA K.M.T., "Performance evaluation of a tracemoisture analyzer based on cavity ringdown spectroscopy: Direct comparison with the NMIJ tracemoisture standard", Sens. Actuat. A: Physical, 165, 2, 2011, 230-238.

[14] NF ISO 5725-1, "Application de la statistique Exactitude (justesse et fidélité) des résultats et méthodes de mesure - Partie 1 : Principes généraux et définitions", 1994. 